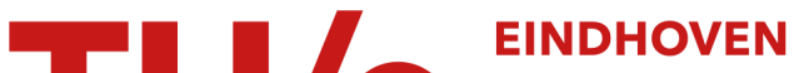 UNIVERSITY OF TECHNOLOGY
}

\section{Characterization of Lipid Nanoparticles Containing lonizable Cationic Lipids Using Design-of-Experiments Approach}

\section{Citation for published version (APA):}

Terada, T., Kulkarni, J. A., Huynh, A., Chen, S., van der Meel, R., Tam, Y. Y. C., \& Cullis, P. R. (2021). Characterization of Lipid Nanoparticles Containing lonizable Cationic Lipids Using Design-of-Experiments Approach. Langmuir, 37(3), 1120-1128. https://doi.org/10.1021/acs.langmuir.0c03039

\section{Document license:}

TAVERNE

DOI:

10.1021/acs.langmuir.0c03039

Document status and date:

Published: 26/01/2021

\section{Document Version:}

Publisher's PDF, also known as Version of Record (includes final page, issue and volume numbers)

\section{Please check the document version of this publication:}

- A submitted manuscript is the version of the article upon submission and before peer-review. There can be important differences between the submitted version and the official published version of record. People interested in the research are advised to contact the author for the final version of the publication, or visit the $\mathrm{DOI}$ to the publisher's website.

- The final author version and the galley proof are versions of the publication after peer review.

- The final published version features the final layout of the paper including the volume, issue and page numbers.

Link to publication

\section{General rights}

Copyright and moral rights for the publications made accessible in the public portal are retained by the authors and/or other copyright owners and it is a condition of accessing publications that users recognise and abide by the legal requirements associated with these rights.

- Users may download and print one copy of any publication from the public portal for the purpose of private study or research.

- You may not further distribute the material or use it for any profit-making activity or commercial gain

- You may freely distribute the URL identifying the publication in the public portal.

If the publication is distributed under the terms of Article 25fa of the Dutch Copyright Act, indicated by the "Taverne" license above, please follow below link for the End User Agreement:

www.tue.nl/taverne

Take down policy

If you believe that this document breaches copyright please contact us at:

openaccess@tue.nl

providing details and we will investigate your claim. 


\section{Characterization of Lipid Nanoparticles Containing lonizable Cationic Lipids Using Design-of-Experiments Approach}

Takeshi Terada,* Jayesh A. Kulkarni, Ariel Huynh, Sam Chen, Roy van der Meel, Yuen Yi C. Tam, and Pieter R. Cullis

Cite This: Langmuir 2021, 37, 1120-1128

Read Online

ACCESS | Llll Metrics \& More | 国 Article Recommendations | sl Supporting Information

ABSTRACT: Lipid nanoparticles (LNPs) containing short-interfering RNA (LNP-siRNA systems) are a promising approach for silencing disease-causing genes in hepatocytes following intravenous administration. LNP-siRNA systems are generated by rapid mixing of lipids in ethanol with siRNA in aqueous buffer ( $\mathrm{pH}$ 4.0) where the ionizable lipid is positively charged, followed by dialysis to remove ethanol and to raise the $\mathrm{pH}$ to 7.4. Ionizable cationic lipids are the critical excipient in LNP systems as they drive entrapment and intracellular delivery. A recent study on the formation of LNP-siRNA systems suggested that ionizable cationic lipids segregate from other lipid components upon charge neutralization to form an amorphous oil droplet in the core of LNPs. This leads to a decrease in intervesicle

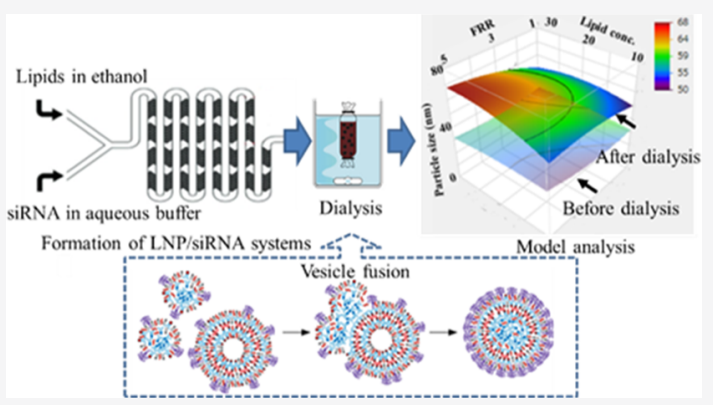
electrostatic repulsion, thereby engendering fusion of small vesicles to form final LNPs of increased size. In this study, we prepared LNP-siRNA systems containing four lipid components (hydrogenated soy phosphatidylcholine, cholesterol, PEG-lipid, and 1,2dioleoyl-3-dimethylammonium propane) by microfluidic mixing. The effects of preparation parameters [lipid concentration, flow rate ratio (FRR), and total flow rate], dialysis process, and complex formation between siRNA and ionizable cationic lipids on the physicochemical properties [siRNA entrapment on the particle size and polydispersity index (PDI)] were investigated using a design of experiments approach. The results for the preparation parameters showed no impact on siRNA encapsulation, but lipid concentration and FRR significantly affected the particle size and PDI. In addition, the effect of FRR on the particle size was suppressed in the presence of anionic polymers such as siRNA as compared to the case of LNPs alone. More intriguingly, unlike empty LNPs, a decrease in the PDI and an increase in the particle size occurred after dialysis in the LNP-siRNA systems. Such changes by dialysis were suppressed at $F R R=1$. These findings provide useful information to guide the development and manufacturing conditions for LNP-siRNA systems.

\section{INTRODUCTION}

Lipid nanoparticles (LNPs) containing ionizable cationic lipids are the most advanced nonviral nucleic acid delivery systems. ${ }^{1,2}$ The ionizable cationic lipid component of LNP-shortinterfering RNA (LNP-siRNA) systems, with an apparent $\mathrm{p} K_{\mathrm{a}}$ of less than 7.0, plays a critical role in siRNA entrapment and endosomal escape within the cell and is also involved in dictating the internal morphology of the LNPs. ${ }^{3-5}$ An established rapid-mixing method for the preparation of LNPsiRNA systems is microfluidics mixing as it affords controlled mixing at the nanoliter scale. ${ }^{7-9}$ LNP-siRNA systems are generated by mixing an ethanolic solution of lipids with siRNA in aqueous buffer at $\mathrm{pH} 4.0$ where ionizable cationic lipids are positively charged. Subsequently, LNP-siRNA suspensions are dialyzed to remove ethanol and to neutralize the $\mathrm{pH}$, leading to LNPs with a relatively neutral surface.

A recent study showed that as the $\mathrm{pH}$ is raised to neutral, an increasing proportion of the ionizable cationic lipids adopts a neutral form, thus decreasing the intervesicle electrostatic repulsion, destabilizing the bilayer structure, and engendering vesicle fusion which leads to an increase in the LNP size. ${ }^{6,10,11}$ As the neutral ionizable lipids partition to the LNP core forming an oil-droplet phase, the particles fuse to compensate for the decreased surface-to-core lipid ratio. Changes of LNPsiRNA physicochemical properties (LNP size, surface composition, and morphology) have been shown to affect the in vivo potency. ${ }^{12-14}$ Therefore, understanding the effect of ionizable cationic lipids on the physicochemical properties of LNP-siRNA systems from a manufacturing perspective is important for robust process development. However, only limited reports are available on the impact of various formulation parameters on the physicochemical parameters of LNP systems.

Received: October 19, 2020

Revised: December 29, 2020

Published: January 13, 2021 

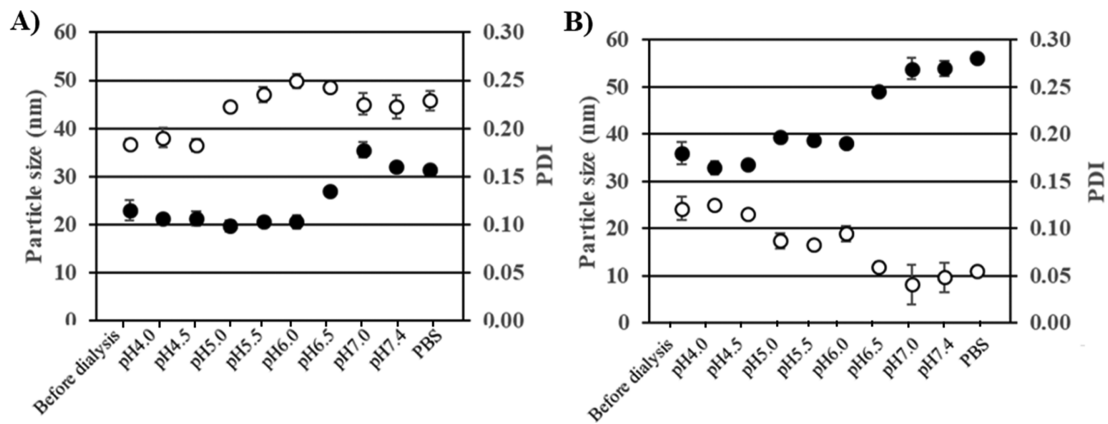

Figure 1. Effect of pH during dialysis on the particle size (filled circle) and PDI (empty circle) of empty LNPs (A) and LNP-siRNA systems (B). Empty LNPs and LNP-siRNA suspensions injected into microfluidics were dialyzed against $25 \mathrm{mM}$ sodium acetate buffer $(\mathrm{pH} 4.0$, $\mathrm{pH} 4.5$, and $\mathrm{pH}$ 5.0), $50 \mathrm{mM}$ 2-( $N$-morpholino)ethanesulfonic acid buffer ( $\mathrm{pH} 5.5, \mathrm{pH} 6.0, \mathrm{pH} 6.5$, and $\mathrm{pH} 7.0$ ), $50 \mathrm{mM}$ HEPES ( $\mathrm{pH} 7.4$ ), and PBS for at least 4 h. Results indicate mean \pm standard deviation $(n=3)$.

In this study, we investigated the effect of preparation parameters [lipid concentration, flow rate ratio (FRR), and total flow rate (TFR)] on the particle size, polydispersity index (PDI), and siRNA entrapment of LNP systems prepared by microfluidics, including before and after dialysis against neutral solution [phosphate-buffered saline (PBS)]. Furthermore, the relationship between the preparation parameters and the properties was evaluated for LNPs with and without siRNA by performing the same procedure for empty LNPs. A design of experiments (DoE) approach was used to systematically evaluate these parameters, resulting in a decreased number of formulation runs. ${ }^{15-17}$ In order to implement a DoE approach, LNPs were prepared using hydrogenated soy phosphatidylcholine (HSPC), cholesterol (Chol), and 1,2-distearoyl-sn-glycero3-phosphoethanol amine- $N$-[methoxy-(polyethylene glycol)2000] (ammonium salt) (PEG-DSPE), which have the added benefit of being clinically approved lipid compounds. In addition, we used, 1,2-dioleoyl-3-dimethylammonium propane (DODAP) that has a reported apparent $\mathrm{p} K_{\mathrm{a}}$ of 6.58. ${ }^{18,19}$ Supporting Information studies were performed to provide insight on the mechanistic effect of manufacturing conditions and formulation on the physicochemical properties.

\section{MATERIALS AND METHODS}

Materials. Lipid HSPC, PEG-DSPE, and DODAP were purchased from Avanti Polar Lipids (Alabaster, AL). Chol and hyaluronic acid (HA) sodium (MW: 8000-15,000) were purchased from SigmaAldrich (St. Louis, MO). The siRNA (siGAPDH) for this study was purchased from Integrated DNA Technologies (San Diego, CA). The siRNA sense and antisense strand sequences are mCmUrCmArUmUrUrCrCrUrGmGrUmArUmGrAmCrArAr CrGrAmAT, rArUmUrCrGrUrUrGmUrCmArUmArCrCrArGrGrArArArUmGrAmGmCmU, respectively.

Preparation of LNPs/LNP-siRNA Systems. LNPs were prepared as previously described. ${ }^{7,20}$ Briefly, lipid components composed of DODAP/Chol/HSPC/PEG-DSPE (50/10/39/1 mol \%) were dissolved in ethanol to a concentration of $10-30 \mathrm{mM}$ total lipid. The aqueous phase consisted of $25 \mathrm{mM}$ sodium acetate $\mathrm{pH} 4$ buffer. Since it has been reported that the $\mathrm{pH}$ of the aqueous phase affects the entrapment of nucleic acids in LNPs, ${ }^{21,22}$ LNPs were prepared at a constant value of $\mathrm{pH} 4.0$ in this study. In the case of LNP-siRNA systems, siRNA was dissolved at a charge ratio of N/P $=3$ in $25 \mathrm{mM}$ sodium acetate $\mathrm{pH} 4$ buffer. The two solutions were mixed through a staggered herringbone micromixer ${ }^{7}$ at a TFR of $1-3 \mathrm{~mL} / \mathrm{min}$ and a FRR of the aqueous and the ethanol phases which was varied from 1 to $5 \mathrm{v} / \mathrm{v}$ (aqueous/ethanol ratio). Unless otherwise specified, the resulting suspension was subsequently dialyzed against at least 1000fold volume of PBS ( $\mathrm{pH} 7.4$ ) overnight (12-14 kD MWCO dialysis tubing, Spectrum Labs, Rancho Dominguez, CA).
Analysis of LNPs. Particle size and PDI analysis of empty LNPs and LNP-siRNA systems were carried out using dynamic light scattering with a Malvern Zetasizer (Worcestershire, UK). In order to avoid the influence of ethanol on the measurement, the concentration of ethanol in the sample was adjusted to be less than $0.5 \%$. Encapsulation efficiency of siRNA was determined using the Quant-iT Ribogreen RNA assay (Life Technologies, Burlington, ON). ${ }^{12}$ Briefly, LNP-siRNA was incubated at $37{ }^{\circ} \mathrm{C}$ for $10 \mathrm{~min}$ in the presence or absence of 1\% Triton X-100 (Sigma-Aldrich, St. Louis, MO) followed by the addition of the ribogreen reagent. The fluorescence intensity (Ex/Em: 480/520 nm) was determined, and the samples treated with Triton X-100 represent total siRNA while untreated samples represent unencapsulated siRNA. Total lipid was determined by measuring the Chol content using the Chol $\mathrm{E}$ assay (Wako Chemicals, Richmond, VA), and the siRNA concentration was determined by measuring the absorbance at $260 \mathrm{~nm} .{ }^{12}$

DOE Approach. The experimental data were analyzed with the statistical software JMP 13 (SAS Institute). ${ }^{23}$ In this DoE approach, the three-factor Box-Behnken design was used to be suitable for second-order models, which was composed of 15 preparation runs. The design is cited as a common experimental design for screening crucial factors. In this design, all factors (lipid concentration, FRR, and TFR) have three levels: low, center, and high. In addition, three center samples were included in this design and used as a source for error estimation. It was important to assign the appropriate level ranges to each of the factors as they should be neither too close nor too far away from each other. This reduces the probability to miss the optimal effect. The levels of each factor were set based on past reports. ${ }^{7,24,25}$ Multiple regression analysis applying a least-squares assessment was performed to identify the preparation parameters (lipid concentration, FRR, and TFR) considered to be crucial for the particle size and PDI of empty LNPs and LNP-siRNA systems. The quadratic model calculated by using multiple regression analysis is described by the following formula:

$$
\begin{aligned}
Y= & \alpha_{0}+\alpha_{1} X_{1}+\alpha_{2} X_{2}+\alpha_{3} X_{3}+\alpha_{4} X_{1} X_{2}+\alpha_{5} X_{1} X_{3}+\alpha_{6} X_{2} X_{3} \\
& +\alpha_{7} X_{1}^{2}+\alpha_{8} X_{2}^{2}+\alpha_{9} X_{3}^{2}
\end{aligned}
$$

where $Y$ is the particle size or PDI, $X$ is a preparation parameter, and $\alpha$ is a regression coefficient. The predictive accuracy of the model was evaluated by using the scatterplot of experimental versus predicted values and the adjusted $R$-squared value $\left(R^{2}\right)$. Analysis of variance was further conducted to identify the statistically significant terms of the model. In addition, the response surface factors were generated using this model to statistically and comprehensively assess the relationship of the parameters to the particle size and PDI. Since cryogenic transmission electron microscopy micrographs obtained for LNPs have been reported to closely correlate with the number-weighted average produced by dynamic light scattering, ${ }^{26}$ the particle size was displayed by number-weighted values, if not specified.

Statistical Analysis. All values were indicated as mean \pm standard deviation. Statistical comparisons between two conditions were 
performed using paired Student's $t$-test. Values of $P<0.05$ were considered to indicate statistical significance.

\section{RESULTS AND DISCUSSION}

Effect of $\mathrm{pH}$ on the Particle Size and PDI of Empty LNPs and LNP-siRNA Systems. LNP-siRNA formed with the use of a microfluidic device are typically dialyzed in a neutral buffer to remove ethanol and raise the $\mathrm{pH}$ to physiological values. A recent study has revealed that in both empty LNP and LNP-siRNA systems, the ionizable lipid DLinKC2-DMA is neutralized during dialysis in PBS $\mathrm{pH} \mathrm{7.4,}$ leading to the formation of a destabilized lipid bilayer structure that promotes particle fusion. ${ }^{6}$ In our DoE approach, we first verified that a similar fusion can be induced in empty LNPs and LNP-siRNA systems containing the ionizable lipid DODAP by measuring the particle size over the range of $\mathrm{pH}$ 4.0 to $\mathrm{pH} 7.4$ compared to PBS (Figure 1). Both empty LNPs and LNP-siRNA systems showed an increase in particle size starting at around $\mathrm{pH} 6.5$, suggesting that particle fusion had occurred. This is expected since the apparent $\mathrm{p} K_{\mathrm{a}}$ of DODAP has been reported to be $6.58,{ }^{18,19}$ and as the $\mathrm{pH}$ is raised above the $\mathrm{p} K_{\mathrm{a}}$ value, more DODAP molecules become neutral.

Multiple Regression Modeling Based on DoE Approach. We next investigated the effects of preparation parameters (lipid concentration, FRR and TFR), dialysis process, and complex formation between siRNA and ionizable cationic lipids on the resulting properties of empty LNP and LNP-siRNA systems using a multiple regression analysis. Instead of a full-factorial design, the Box-Behnken approach was used as it was suited to create a quadratic model. A threefactor Box-Behnken design is almost rotatable, which means that all design points are at the same distance from the center of the design. Such a design lends itself to aptly create a response surface plot as the prediction error is the same for all design points. ${ }^{27}$ The statistical approach using DoE to design the optimal properties of LNPs has been investigated in many studies, and the usefulness of this approach has been demonstrated in the development of LNP-related product formulations. ${ }^{8,17,28}$

In this DoE study, the preparation parameters are shown in Table 1. Since siRNA encapsulation values showed approximately $90 \%$ in all LNP-siRNA systems (Supporting Information, Figure S1), the particle size and PDI were selected as physicochemical properties to be evaluated further in this study. First, the accuracy of the multiple regression equations constructed by this design was evaluated. As shown in the scatterplots of experimental versus predicted values (Supporting Information, Figure S2), the coefficients of determination adjusted by the degrees of freedom $\left(R^{2}\right)$ were high. The $R^{2}$ values for particle size and PDI of empty LNPs before dialysis were 0.94 and 0.99 , respectively, whereas these values after dialysis were 0.99 and 0.92 , respectively. Similarly, the $R^{2}$ values for particle size and PDI of siRNA-containing systems before dialysis were 0.96 and 0.95 , respectively, and 0.91 and 0.95 , respectively, after dialysis. Additionally, the mean of the experimental data (blue line) fell outside the bounds of the $95 \%$ confidence area (pink area), indicating that the overall regression model was statistically significant.

Evaluation of Statistically Significant Preparation Parameters. We then evaluated this data to further understand the impact of lipid concentration, FRR, and TFR on the particle size and PDI of these systems. As detailed in the methods, the three parameters tested provide for nine variables
Table 1. DoEs for DoE-Based Microfluidic Flow Settings ${ }^{a}$

$\begin{array}{cccc}\text { run no. } & \text { lipid conc. }(\mathrm{mM}) & \text { FRR (vol/vol) } & \text { TFR }(\mathrm{mL} / \mathrm{min}) \\ 1 & 20 & 1 & 1 \\ 2 & 20 & 5 & 1 \\ 3 & 20 & 1 & 3 \\ 4 & 20 & 5 & 3 \\ 5 & 10 & 1 & 2 \\ 6 & 30 & 1 & 2 \\ 7 & 10 & 5 & 2 \\ 8 & 30 & 5 & 2 \\ 9 & 10 & 3 & 1 \\ 10 & 10 & 3 & 3 \\ 11 & 30 & 3 & 1 \\ 12 & 30 & 3 & 3 \\ 13 & 20 & 3 & 2 \\ 14 & 20 & 3 & 2 \\ 15 & 20 & 3 & 2\end{array}$

${ }^{a}$ Different lipid concentrations, FRR, and TFR used for the preparation of each empty LNP and LNP-siRNA system in this study are represented.

that affect the outcomes (particle size and PDI). It can be seen from Table 2 that FRR and FRR $\times$ FRR showed a remarkable effect $(P<0.01)$ on the particle size and PDI of both empty LNPs and LNP-siRNA systems. Previous work using LNPs composed of 1,2-dioleoyl-3-trimethylammonium-propane and 1,2-dioleoyl-sn-glycero-3-phosphoethanolamine has also reported that FRR in the range of 1 to 5 significantly affected the particle size and PDI, ${ }^{8}$ which is in agreement with the results of this study. This highlights the importance of the ethanol-to-aqueous ratio when controlling the physical properties of LNP systems produced by microfluidics. Furthermore, in the case of LNP-siRNA systems, the lipid concentration showed a significant effect on the particle size and PDI. Since the charge ratio $(\mathrm{N} / \mathrm{P})$ in the prepared LNP-siRNA systems was fixed at 3 , the concentration of siRNA was also modified as to maintain a constant lipid-to-siRNA ratio. Therefore, it is expected that as the physical characteristics of LNP-siRNA systems are affected by the local concentration of DODAP/ siRNA binding at the region where the two fluids mix in a microfluidics device, higher concentrations of particles (in the presence of the solvent) lead to increased and unintended particle fusion.

Model Analysis against the Properties of Empty LNPs and LNP-siRNA Systems. The impact on the statistically significant effect of preparation parameters (lipid concentration, FRR, and TFR) on the particle size and PDI of empty LNPs and LNP-siRNA systems before and after dialysis was further investigated in a resonance surface model using our DoE analysis.

The response surface factors against the particle size of empty LNPs and LNP-siRNA systems before and after dialysis are shown in Figures 2 and 3, respectively. An overall trend of increase in particle size following dialysis was observed with both empty LNPs and LNP-siRNA systems. As the FRR was increased (i.e., more aqueous), a reduction in the particle size on both empty LNPs and LNP-siRNA systems was detected. This is likely because FRR strongly affects the polarity throughout the chamber as well as the final ethanol concentration, resulting in the change of exchange rates for individual lipid molecules. In the studies using hydrodynamic flow-focusing techniques, the increase in the FRR has been 
Table 2. Effect of Each Factor against PDI and Particle Size of Empty LNPs and LNP-siRNA Systems

\begin{tabular}{|c|c|c|c|c|c|c|c|c|}
\hline \multirow[b]{4}{*}{ factors } & \multicolumn{8}{|c|}{$P$ value $($ prob $>[t])$} \\
\hline & \multicolumn{4}{|c|}{ empty LNPs } & \multicolumn{4}{|c|}{ LNP-siRNA systems } \\
\hline & \multicolumn{2}{|c|}{ particle size } & \multicolumn{2}{|c|}{ PDI } & \multicolumn{2}{|c|}{ particle size } & \multicolumn{2}{|c|}{ PDI } \\
\hline & before dialysis & after dialysis & before dialysis & after dialysis & before dialysis & after dialysis & before dialysis & after dialysis \\
\hline lipid conc. & 0.044 & 0.879 & 0.187 & 0.271 & 0.001 & 0.025 & 0.018 & 0.040 \\
\hline FRR & $<0.001$ & $<0.001$ & $<0.001$ & 0.009 & $<0.001$ & 0.005 & $<0.001$ & $<0.001$ \\
\hline TFR & 0.138 & 0.923 & 0.154 & 0.181 & 0.013 & 0.739 & 0.325 & 0.660 \\
\hline lipid conc. $\times$ lipid conc. & 0.839 & 0.482 & 0.005 & 0.704 & 0.191 & 0.054 & 0.167 & 0.884 \\
\hline$F R R \times F R R$ & $<0.001$ & 0.006 & $<0.001$ & 0.004 & 0.025 & 0.117 & 0.004 & 0.006 \\
\hline $\mathrm{TFR} \times \mathrm{TFR}$ & 0.452 & 0.599 & 0.036 & 0.077 & 0.220 & 0.462 & 0.972 & 0.944 \\
\hline lipid conc. $\times$ FRR & 0.083 & 0.422 & $<0.001$ & 0.032 & 0.257 & 0.099 & 0.136 & 0.044 \\
\hline lipid conc. $\times$ TFR & 0.874 & 0.793 & 0.459 & 0.969 & 0.115 & 0.680 & 0.852 & 0.973 \\
\hline $\mathrm{FRR} \times \mathrm{TFR}$ & 0.563 & 0.074 & 0.022 & 0.371 & 0.675 & 0.082 & 0.876 & 0.618 \\
\hline
\end{tabular}
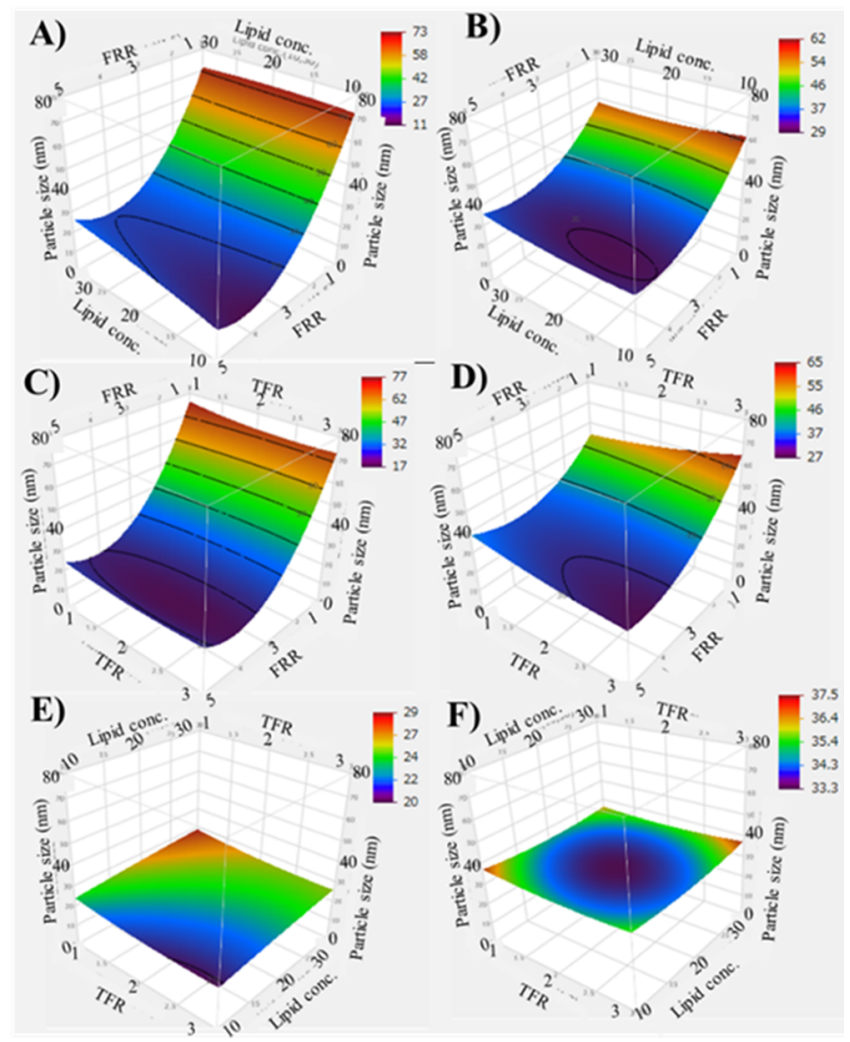

Figure 2. Response surface factors against particle size of empty LNPs before and after dialysis: the effect of lipid concentration $(\mathrm{mM})$ and FRR ( vol/vol) on the particle size before dialysis at TFR $(\mathrm{mL} / \mathrm{min})=$ 2 (A), the effect of lipid concentration and FRR on the particle size after dialysis at TFR $=2(\mathrm{~B})$, the effect of FRR and TFR on the particle size before dialysis at lipid concentration $=20(\mathrm{C})$, the effect of FRR and TFR on the particle size after dialysis at lipid concentration $=20(\mathrm{D})$, the effect of lipid concentration and TFR on the particle size before dialysis at FRR $=3(\mathrm{E})$, and the effect of lipid concentration and TFR on the particle size after dialysis at FRR $=3(\mathrm{~F})$.

reported to decrease the liposome particle size, ${ }^{29,30}$ similar to what was observed in this study. On the other hand, our mathematical model showed that lipid concentration influenced (with statistical significance) the particle size of LNPsiRNA systems but not empty LNPs (Table 2). In the model analysis, it was observed that the particle size of LNP-siRNA systems was affected by the FRR and lipid concentration and
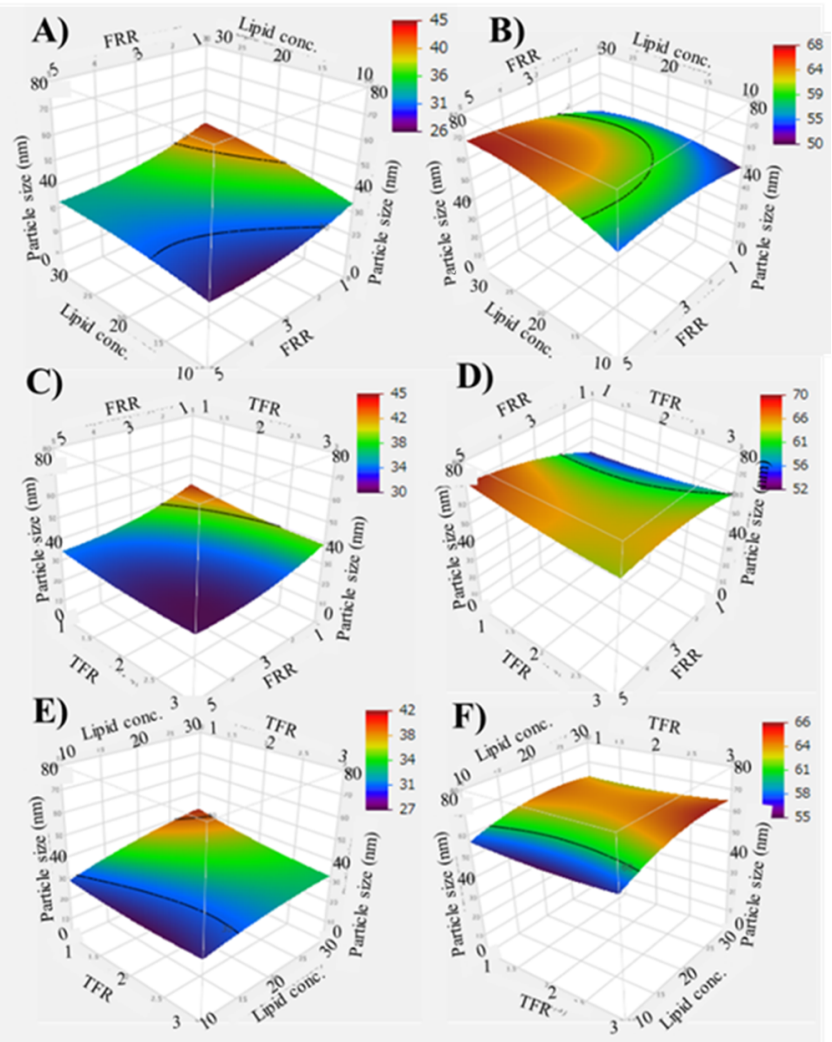

Figure 3. Response surface factors against particle size of LNP-siRNA systems before and after dialysis: the effect of lipid concentration (mM) and FRR ( $\mathrm{vol} / \mathrm{vol})$ on the particle size before dialysis at TFR $(\mathrm{mL} / \mathrm{min})=2(\mathrm{~A})$, the effect of lipid concentration and FRR on the particle size after dialysis at TFR $=2(\mathrm{~B})$, the effect of FRR and TFR on the particle size before dialysis at lipid concentration $=20(\mathrm{C})$, the effect of FRR and TFR on the particle size after dialysis at lipid concentration $=20(\mathrm{D})$, the effect of lipid concentration and TFR on particle size before dialysis, at FRR $=3(\mathrm{E})$, and the effect of lipid concentration and TFR on the particle size after dialysis at FRR $=3$ (F).

confirmed that the particle size will likely increase if a low FRR and a high lipid concentration are used.

Figures 4 and 5 show the response surface factors against the PDI of empty LNPs and LNP-siRNA systems before and after dialysis. It has been reported that FRR is a key parameter to control the PDI of LNPs because the diffusion rate in microfluidics is reduced at a low FRR. ${ }^{29,31}$ Increased FRR (to 


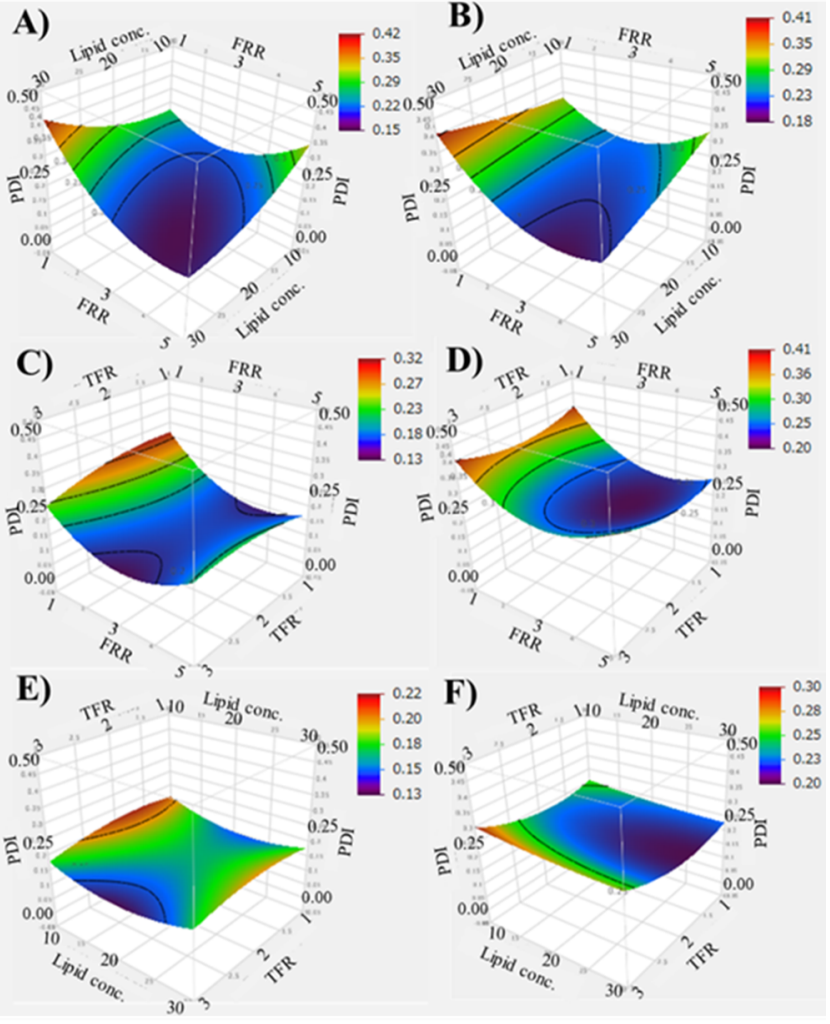

Figure 4. Response surface factors against PDI of empty LNPs before and after dialysis: the effect of lipid concentration $(\mathrm{mM})$ and FRR $(\mathrm{vol} / \mathrm{vol})$ on PDI before dialysis at TFR $(\mathrm{mL} / \mathrm{min})=2(\mathrm{~A})$, the effect of lipid concentration and FRR on PDI after dialysis at TFR $=2$ (B), the effect of FRR and TFR on PDI before dialysis at lipid concentration $=20(\mathrm{C})$, the effect of FRR and TFR on PDI after dialysis at lipid concentration $=20$ (D), the effect of lipid concentration and TFR on PDI before dialysis at FRR $=3(\mathrm{E})$, and the effect of lipid concentration and TFR on PDI after dialysis at FRR $=3(\mathrm{~F})$.

3) was associated with a decreased PDI in both empty LNPs and LNP-siRNA systems. However, in the range of 3-5 FRR, no impact on PDI was observed. In addition, lipid concentration was found to have a greater impact on the PDI of LNP-siRNA systems than on that of empty LNPs, and there was a tendency for the PDI to increase with increasing lipid concentration. Interestingly, a reduction in the PDI was observed following dialysis in LNP-siRNA systems.

Comparative Evaluation of DoE Results for Empty LNPs and LNP-siRNA Systems before and after Dialysis. The experimental results of this DoE approach (outlined in Table 1) are shown in Figure 6 to compare changes in the particle size and PDI before and after dialysis between empty LNPs and LNP-siRNA systems. Two different trends were identified between empty LNPs and LNP-siRNA systems. First, LNP-siRNA systems showed substantial reductions in the PDI following dialysis, while the PDI of empty LNPs generally remained the same or slightly increased following dialysis. Furthermore, the increase in the particle size that occurred after dialysis was much higher with LNP-siRNA systems than with empty LNPs. This observation is consistent with previous data that showed loaded systems (at FRR $=3$ ) display generally larger sizes than the unloaded formulation ${ }^{6}$ and that the presence of siRNA should contribute to increasing particle size.

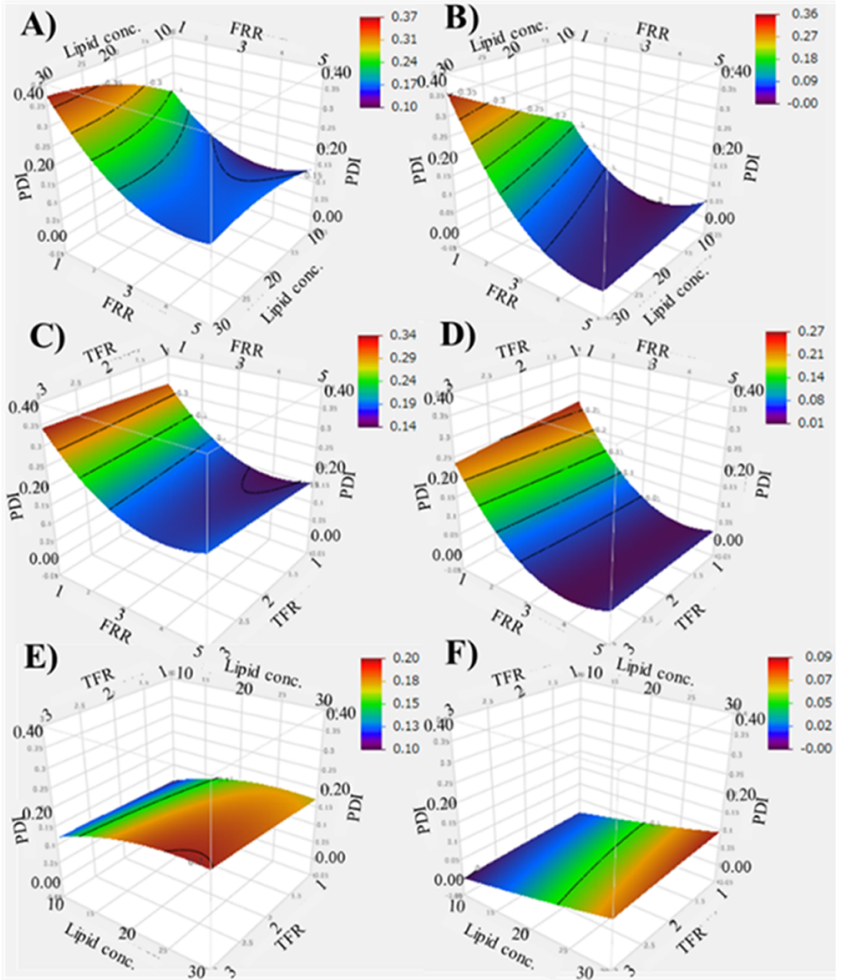

Figure 5. Response surface factors against PDI of LNP-siRNA systems before and after dialysis: the effect of lipid concentration $(\mathrm{mM})$ and FRR (vol/vol) on PDI before dialysis at TFR $(\mathrm{mL} / \mathrm{min})=$ 2 (A), the effect of lipid concentration and FRR on PDI after dialysis at TFR $=2(\mathrm{~B})$, the effect of FRR and TFR on PDI before dialysis at lipid concentration $=20(\mathrm{C})$, the effect of FRR and TFR on PDI after dialysis at lipid concentration $=20$ (D), the effect of lipid concentration and TFR on PDI before dialysis at FRR $=3(\mathrm{E})$, and the effect of lipid concentration and TFR on PDI after dialysis at FRR $=3(\mathrm{~F})$.

The second trend related to the change in particle size and PDI as a result of FRR. Empty LNPs displayed a similar small particle size at FRR = 3 and 5; however, the particle sizes (and PDI) before and after dialysis were much larger at FRR $=1$. In contrast, for LNP-siRNA, the particle sizes stayed relatively consistent before dialysis regardless of FRR. A similar observation was made for the post-dialysis samples, where all formulations displayed similar sizes that are generally larger than their predialysis counterparts. This indicates that the presence of anionic cargo such as siRNA limits the rearrangement of lipids. Consistent with previous observations, ${ }^{6,11}$ we believe that the dramatic differences for empty LNPs were a result of substantial lipid reorganization.

Effect of $\mathrm{pH}$ Neutralization in the Presence or Absence of Ethanol. LNP-siRNA suspensions prepared at $\mathrm{FRR}=1$ (with $50 \%$ ethanol $\mathrm{v} / \mathrm{v}$ ) showed little change in the particle size and PDI before and after dialysis. In order to study the effect of $\mathrm{pH}$ neutralization in the presence of ethanol on the particle size and PDI of LNP-siRNA, two formulation processes were tested. LNP-siRNA produced at FRR $=3$ and 1 were collected and neutralized by (1) dialysis against PBS or (2) by injection into microfluidics with $50 \mathrm{mM} \mathrm{4-(2-}$ hydroxyethyl)-1-piperazineethanesulfonic acid (HEPES) buffer ( $\mathrm{pH}$ 8.0) containing $200 \mathrm{mM}$ sodium chloride followed by dialysis. The composition of the HEPES buffer was adjusted to show a salt concentration and $\mathrm{pH}$ similar to those of $\mathrm{PBS}$ after 
A) Empty LNPs

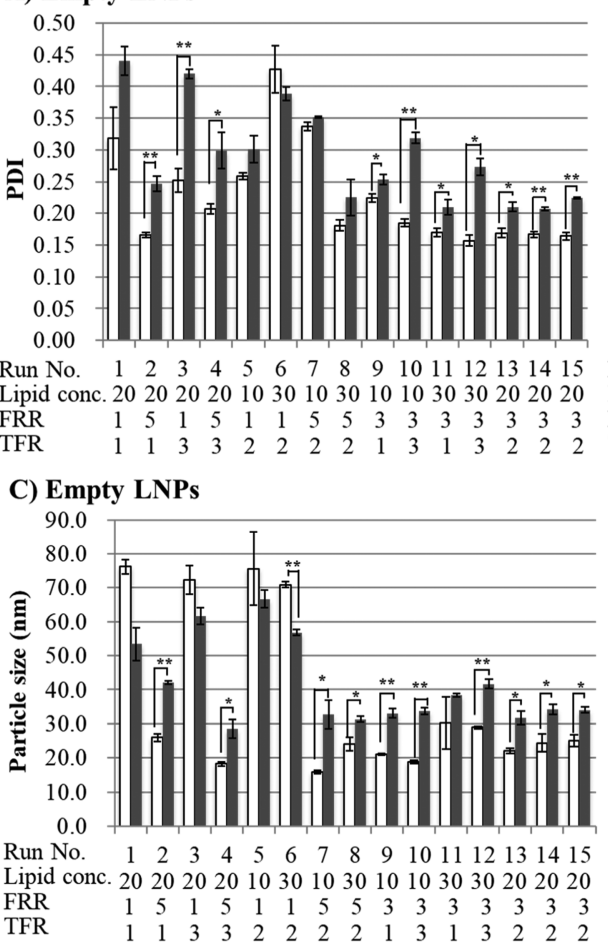

B) LNP-siRNA systems

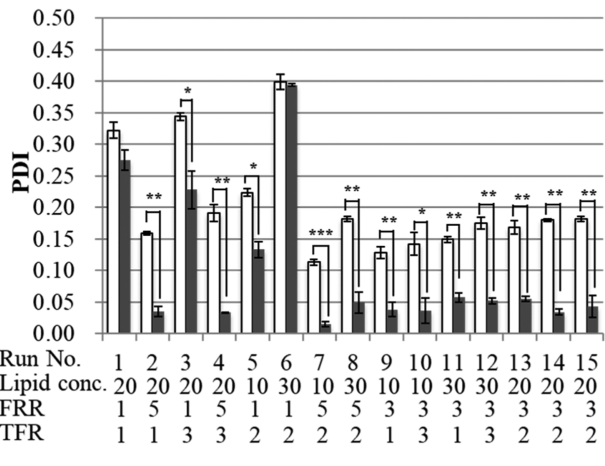

D) LNP-siRNA systems

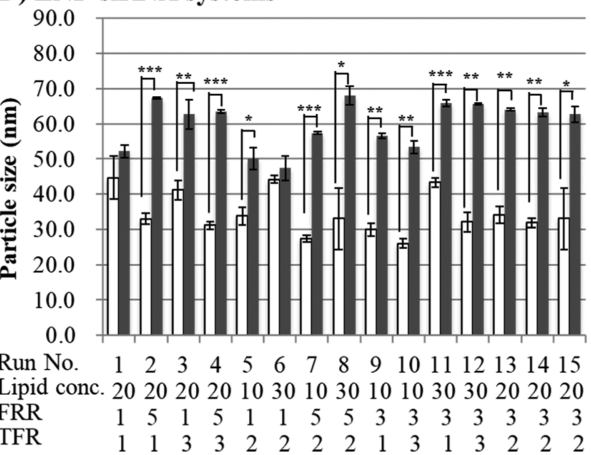

Figure 6. Comparative evaluation of DoE results for empty LNPs and LNP-siRNA systems before (white bar) and after dialysis (gray bar): PDI before and after dialysis of empty LNPs (A) and LNP-siRNA systems (B) and particle size before and after dialysis of empty LNPs (C) and LNPsiRNA systems (D). For formulation parameters of each run, please refer to Table 1 . Results indicate mean \pm standard deviation $(n=3)$. $* * P<$ $0.05, * * P<0.01$, and $* * * P<0.001$.
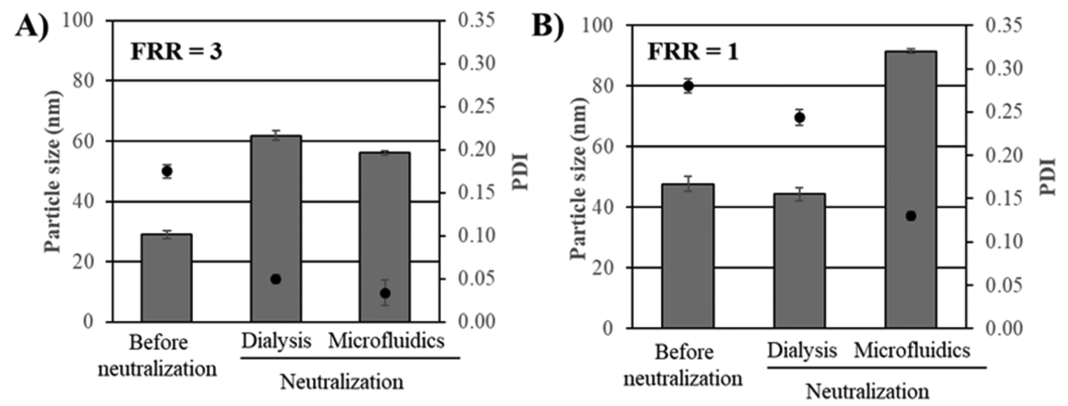

Figure 7. Effect of ethanol content on the change of particle size (solid bar) and PDI (closed circle) in different pH neutralization methods. LNPsiRNA suspensions were prepared under the condition of FRR $=3(\mathrm{~A})$ and 1 (B) by microfluidics. Each suspension was divided to neutralize by

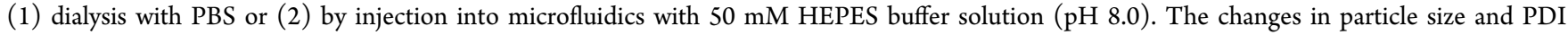
before and after neutralization of the suspension prepared at FRR $=3$ and 1 were compared for different neutralization methods. Results indicate mean \pm standard deviation $(n=3)$.

the injection into the microfluidics. As shown in Figure 7A, LNP-siRNA prepared at FRR $=3$ showed an increase in particle size and a decrease in PDI after neutralization, but there was no difference between the two $\mathrm{pH}$ neutralization methods. On the other hand, as shown in Figure 7B, in LNPsiRNA suspensions with $F R R=1$, a significant increase in the particle size and a decrease in the PDI were observed after $\mathrm{pH}$ neutralization using the microfluidic mixer. This relationship between the FRR and particle morphology is consistent with similar observations for particles composed of DSPC/Chol/ PEG-DSPE (52/45/3 mol \%) prepared using the same technique at FRRs of 1 and 3 (Supporting Information, Figure S3). It suggests that higher FRRs produce larger particle sizes even when the protonation states of ionizable lipids are not convoluting factors to the size and morphology. High ethanol contents provide an environment with relatively high viscosity, osmolality, and lipid solubility, which are supportive of uncontrolled particle fusion. In addition, it is likely that the LNP metamorphosis that occurs as the $\mathrm{pH}$ is neutralized is a shear-sensitive process leading to a particle size increase. Although further studies are needed, our findings suggest that the method of $\mathrm{pH}$ neutralization process in manufacturing is important to obtain LNP-siRNA with desirable properties (defined particle sizes, low polydispersity indices, and high entrapment).

Effect of the Binding of siRNA with DODAP on the Particle Size of siRNA-LNP Systems. The increase in the particle size with decreasing FRR (i.e., more ethanol) was suppressed in the presence of siRNA as compared to the case of empty LNPs. Therefore, it is considered that small vesicles 

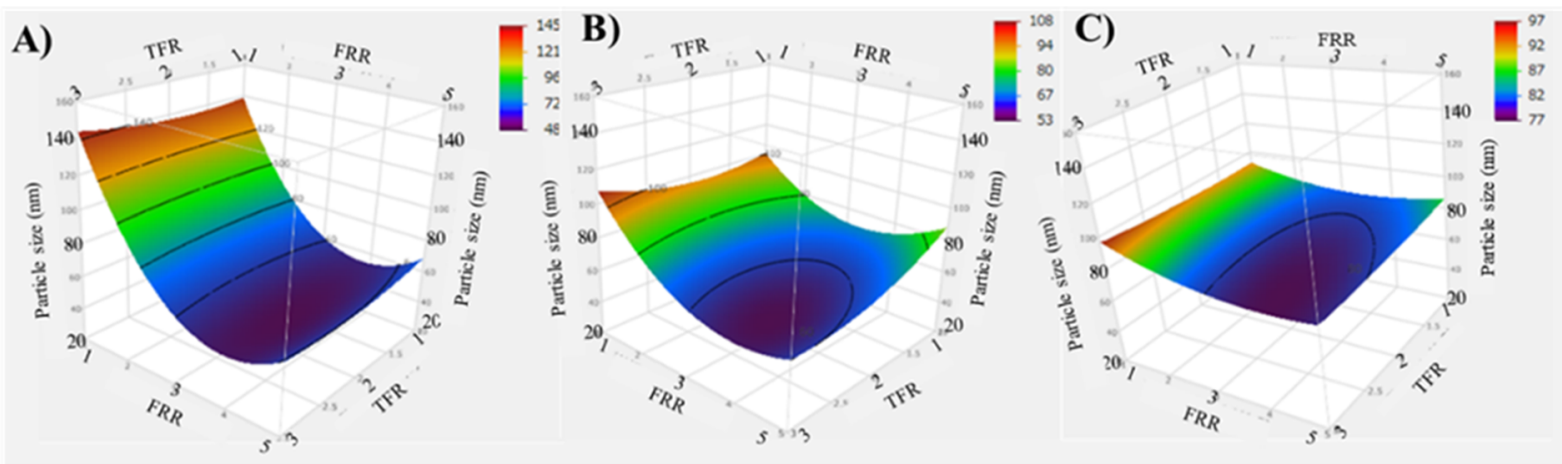

Figure 8. Response surface factors against particle size of empty LNPs (A), LNP-HA complexes (B), and LNP-siRNA systems (C) after dialysis: the effect of FRR ( vol/vol) and TFR $(\mathrm{mL} / \mathrm{min})$ on the particle size at lipid concentration $=20 \mathrm{mM}$. In the preparation of LNP-HA complexes, HA was dissolved at a charge ratio of DODAP nitrogen/HA carboxylic acid $(\mathrm{N} / \mathrm{COOH})=3$ in acetate buffer.

which contain siRNA between closely apposed lipid monolayers are not affected by the ethanol concentration because of their low solubility in ethanol. In addition to this, we have previously shown that "free" ionizable lipid (i.e., not interacting with nucleic acid) contributes to particle rearrangement and that ionizable lipid interacting with nucleic acid does not support rearrangement. With high ethanol ratios (low FRR), empty LNPs showed a remarkable increase of particle size, likely due to increased solubility of all lipid components in the high ethanol content solution.

In order to show that the formation of small vesicles with anionic polymers is related to the change in particle size with low FRR, complexes with HA with molecular weight similar to that of siRNA were prepared, and the particle size was evaluated. HA has previously been reported to form complexes with cationic liposomes in a manner similar to nucleic acids, ${ }^{32}$ although the acidity of its carboxylic acid is relatively lower than that of phosphoric acid in siRNA. The experiments were performed using the same DoE approach as in the comparative studies of empty LNP with LNP-siRNA systems, and the results were analyzed as resonance surface modeling based on $z$-average values. As expected, LNP-HA systems (LNP containing HA) demonstrated that the degree of change in particle size caused by FRR was smaller than that of empty LNPs but larger than that of LNP-siRNA systems, suggesting that the binding affinity of small vesicles with anionic cargos plays a significant role in the formulation of the LNP complexes (Figure 8). Further studies are required to determine how different types of anionic cargos (modified nucleic acids or peptides) influence nanoparticle formation.

\section{CONCLUSIONS}

In this paper, since little has been reported on the evaluation of the effect of manufacturing parameters on the particle size by tracing from microfluidics to dialysis, the effect of microfluidics parameters and subsequent dialysis on the physicochemical properties of empty LNPs and LNP-siRNA systems was elucidated by statistical evaluation using a DoE approach. The preparation parameters (lipid concentration, FRR, and TFR) were evaluated, and lipid concentration and FRR were identified as the critical process parameters to be monitored in order to achieve the desired and robust LNP-siRNA particles. We believe that such a study contributes critical and useful information to the generation of a design space for manufacturing parameters in the process development of LNPsiRNA systems. Furthermore, we demonstrated that, in each manufacturing step from rapid mixing to dialysis, the presence of siRNA dramatically impacts the physicochemical properties of LNP-siRNA systems. The results and discussions above are expected to enable robust commercial production from lab-ona-chip scale not only for LNP-siRNA systems but also for new formulations of LNP systems with other anionic cargos such as mRNA and DNA.

\section{ASSOCIATED CONTENT}

\section{SI Supporting Information}

The Supporting Information is available free of charge at https://pubs.acs.org/doi/10.1021/acs.langmuir.0c03039.

Encapsulation efficiency of siRNA/LNP systems prepared by microfluidics in the DoE approach; scatterplots of experimental vs predicted values modeled by multiple regression based on DoE for PDI and particle size; and effect of FRR on the morphology of particles composed of DSPC/Chol/PEG-DSPE (52/45/3 mol \%)] (PDF)

\section{AUTHOR INFORMATION}

\section{Corresponding Author}

Takeshi Terada - Department of Biochemistry and Molecular Biology, University of British Columbia, Vancouver, British Columbia V6T 1Z3, Canada; (o orcid.org/0000-00029215-6744; Email: terada.takeshi@mk.mt-pharma.co.jp

\section{Authors}

Jayesh A. Kulkarni - Department of Biochemistry and Molecular Biology, University of British Columbia, Vancouver, British Columbia V6T 1Z3, Canada; (1) orcid.org/0000-0002-3622-6998

Ariel Huynh - Department of Biochemistry and Molecular Biology, University of British Columbia, Vancouver, British Columbia V6T 1Z3, Canada

Sam Chen - Department of Biochemistry and Molecular Biology, University of British Columbia, Vancouver, British Columbia V6T 1Z3, Canada

Roy van der Meel - Department of Biochemistry and Molecular Biology, University of British Columbia, Vancouver, British Columbia V6T 1Z3, Canada

Yuen Yi C. Tam - Department of Biochemistry and Molecular Biology, University of British Columbia, Vancouver, British Columbia V6T 1Z3, Canada

Pieter R. Cullis - Department of Biochemistry and Molecular Biology, University of British Columbia, Vancouver, British 
Columbia V6T 1Z3, Canada; ๑ orcid.org/0000-00019586-2508

Complete contact information is available at: https://pubs.acs.org/10.1021/acs.langmuir.0c03039

\section{Author Contributions}

The manuscript was written through contributions of all authors. All authors have given approval to the final version of the manuscript.

\section{Notes}

The authors declare no competing financial interest.

\section{ACKNOWLEDGMENTS}

This project was partially funded by Mitsubishi Tanabe Pharma Corporation. P.R.C. is funded by a Canadian Institutes of Health Research Foundation grant (FDN 148469). P.R.C. is also the CEO and Scientific Director of the NanoMedicines Innovation Network (NMIN), a National Centers of Excellence of Canada (NCE). J.A.K. is supported by a NMIN Postdoctoral Award in Gene Therapy.

\section{ABBREVIATIONS}

LNPs, lipid nanoparticles; FRR, flow rate ratio; TFR, total flow rate; DoE, design of experiments; PDI, polydispersity index; HSPC, hydrogenated soy phosphatidylcholine; PEG-DSPE, 1,2-distearoyl-sn-glycero-3-phosphoethanol amine- $N$-[methoxy-(polyethylene glycol)-2000]; Chol, cholesterol; DODAP, 1,2-dioleoyl-3-dimethylammonium propane; SHM, staggered herringbone micromixer; PBS, phosphate buffered saline; HA, hyaluronic acid; DOPE, 1,2-dioleoyl-sn-glycero-3phosphoethanolamine; DOTAP, 1,2-dioleoyl-3-trimethylammonium-propane

\section{REFERENCES}

(1) Akinc, A.; Maier, M. A.; Manoharan, M.; Fitzgerald, K.; Jayaraman, M.; Barros, S.; Ansell, S.; Du, X.; Hope, M. J.; Madden, T. D.; Mui, B. L.; Semple, S. C.; Tam, Y. K.; Ciufolini, M.; Witzigmann, D.; Kulkarni, J. A.; van der Meel, R.; Cullis, P. R. The Onpattro story and the clinical translation of nanomedicines containing nucleic acidbased drugs. Nat. Nanotechnol. 2019, 14, 1084-1087.

(2) Kulkarni, J. A.; Witzigmann, D.; Chen, S.; Cullis, P. R.; van der Meel, R. Lipid Nanoparticle Technology for Clinical Translation of siRNA Therapeutics. Acc. Chem. Res. 2019, 52, 2435-2444.

(3) Tam, Y.; Chen, S.; Cullis, P. Advances in Lipid Nanoparticles for siRNA Delivery. Pharmaceutics 2013, 5, 498-507.

(4) Semple, S. C.; Akinc, A.; Chen, J.; Sandhu, A. P.; Mui, B. L.; Cho, C. K.; Sah, D. W. Y.; Stebbing, D.; Crosley, E. J.; Yaworski, E.; Hafez, I. M.; Dorkin, J. R.; Qin, J.; Lam, K.; Rajeev, K. G.; Wong, K. F.; Jeffs, L. B.; Nechev, L.; Eisenhardt, M. L.; Jayaraman, M.; Kazem, M.; Maier, M. A.; Srinivasulu, M.; Weinstein, M. J.; Chen, Q.; Alvarez, R.; Barros, S. A.; De, S.; Klimuk, S. K.; Borland, T.; Kosovrasti, V.; Cantley, W. L.; Tam, Y. K.; Manoharan, M.; Ciufolini, M. A.; Tracy, M. A.; de Fougerolles, A.; MacLachlan, I.; Cullis, P. R.; Madden, T. D.; Hope, M. J. Rational design of cationic lipids for siRNA delivery. Nat. Biotechnol. 2010, 28, 172-176.

(5) Jayaraman, M.; Ansell, S. M.; Mui, B. L.; Tam, Y. K.; Chen, J.; Du, X.; Butler, D.; Eltepu, L.; Matsuda, S.; Narayanannair, J. K.; Rajeev, K. G.; Hafez, I. M.; Akinc, A.; Maier, M. A.; Tracy, M. A.; Cullis, P. R.; Madden, T. D.; Manoharan, M.; Hope, M. J. Maximizing the Potency of siRNA Lipid Nanoparticles for Hepatic Gene Silencing In Vivo. Angew. Chem., Int. Ed. 2012, 51, 8529-8533.

(6) Kulkarni, J. A.; Darjuan, M. M.; Mercer, J. E.; Chen, S.; van der Meel, R.; Thewalt, J. L.; Tam, Y. Y. C.; Cullis, P. R. On the formation and morphology of lipid nanoparticles containing ionizable cationic lipids and siRNA. ACS Nano 2018, 12, 4787-4795.
(7) Belliveau, N. M.; Huft, J.; Lin, P. J.; Chen, S.; Leung, A. K.; Leaver, T. J.; Wild, A. W.; Lee, J. B.; Taylor, R. J.; Tam, Y. K.; Hansen, C. L.; Cullis, P. R. Microfluidic Synthesis of Highly Potent Limit-size Lipid Nanoparticles for In Vivo Delivery of siRNA. Mol. Ther.-Nucleic Acids 2012, 1, No. e37.

(8) Kastner, E.; Kaur, R.; Lowry, D.; Moghaddam, B.; Wilkinson, A.; Perrie, Y. High-throughput manufacturing of size-tuned liposomes by a new microfluidics method using enhanced statistical tools for characterization. Int. J. Pharm. 2014, 477, 361-368.

(9) Song, Y.; Hormes, J.; Kumar, C. S. S. R. Microfluidic synthesis of nanomaterials. Small 2008, 4, 698-711.

(10) Ramezanpour, M.; Schmidt, M. L.; Bodnariuc, I.; Kulkarni, J. A.; Leung, S. S. W.; Cullis, P. R.; Thewalt, J. L.; Tieleman, D. P. Ionizable amino lipid interactions with POPC: implications for lipid nanoparticle function. Nanoscale 2019, 11, 14141-14146.

(11) Kulkarni, J. A.; Witzigmann, D.; Leung, J.; van der Meel, R.; Zaifman, J.; Darjuan, M. M.; Grisch-Chan, H. M.; Thöny, B.; Tam, Y. Y. C.; Cullis, P. R. Fusion-dependent formation of lipid nanoparticles containing macromolecular payloads. Nanoscale 2019, 11, 90239031.

(12) Chen, S.; Tam, Y. Y. C.; Lin, P. J. C.; Sung, M. M. H.; Tam, Y. K.; Cullis, P. R. Influence of Particle Size on the in Vivo Potency of Lipid Nanoparticle Formulations of siRNA. J. Controlled Release 2016, 235, 236-244.

(13) Koltover, I.; Salditt, T.; Rädler, J. O.; Safinya, C. R. An inverted hexagonal phase of cationic liposome-DNA complexes related to DNA release and delivery. Science 1998, 281, 78-81.

(14) Kim, H.; Leal, C. Cuboplexes: Topologically active siRNA delivery. ACS Nano 2015, 9, 10214-10226.

(15) Mandenius, C.-F.; Brundin, A. Bioprocess optimization using design-of-experiments methodology. Biotechnol. Prog. 2008, 24, 1191-1203.

(16) Yu, L. X.; Amidon, G.; Khan, M. A.; Hoag, S. W.; Polli, J.; Raju, G. K.; Woodcock, J. Understanding pharmaceutical quality by design. AAPS. J. 2014, 16, 771-783.

(17) Sedighi, M.; Sieber, S.; Rahimi, F.; Shahbazi, M.-A.; Rezayan, A. H.; Huwyler, J.; Witzigmann, D. Rapid Optimization of Liposome Characteristics Using a Combined Microfluidics and design-ofexperiment Approach. Drug Delivery Transl. Res. 2019, 9, 404-413.

(18) Bailey, A. L.; Cullis, P. R. Modulation of Membrane Fusion by Asymmetric Transbilayer Distributions of Amino Lipids. Biochemistry 1994, 33, 12573-12580.

(19) Cullis, P. R.; Hope, M. J. Lipid Nanoparticle Systems for Enabling Gene Therapies. Mol. Ther. 2017, 25, 1467-1475.

(20) Leung, A. K. K.; Hafez, I. M.; Baoukina, S.; Belliveau, N. M.; Zhigaltsev, I. V.; Afshinmanesh, E.; Tieleman, D. P.; Hansen, C. L.; Hope, M. J.; Cullis, P. R. Lipid Nanoparticles Containing siRNA Synthesized by Microfluidic Mixing Exhibit an Electron-Dense Nanostructured Core. J. Phys. Chem. C 2012, 116, 18440-18450.

(21) Semple, S. C.; Klimuk, S. K.; Harasym, T. O.; Dos Santos, N.; Ansell, S. M.; Wong, K. F.; Maurer, N.; Stark, H.; Cullis, P. R.; Hope, M. J.; Scherrer, P. Efficient encapsulation of antisense oligonucleotides in lipid vesicles using ionizable aminolipids: formation of novel small multilamellar vesicle structures. Biochim. Biophys. Acta 2001, $1510,152-166$.

(22) Jeffs, L. B.; Palmer, L. R.; Ambegia, E. G.; Giesbrecht, C.; Ewanick, S.; MacLachlan, I. A scalable, extrusion-free method for efficient liposomal encapsulation of plasmid DNA. Pharm. Res. 2005, 22, 362-372.

(23) Goupy, J.; Creighton, L. Introduction to Design of Experiments with JMP Examples, 3rd ed.; SAS Institute Inc.: Cary, NC, 2007.

(24) Kastner, E.; Verma, V.; Lowry, D.; Perrie, Y. Microfluidiccontrolled manufacture of liposomes for the solubilisation of a poorly water soluble drug. Int. J. Pharm. 2015, 485, 122-130.

(25) Cheung, C. C. L.; Al-Jamal, W. T. Sterically stabilized liposomes production using staggered herringbone micromixer: Effect of lipid composition and PEG-lipid content. Int. J. Pharm. 2019, 566, 687-696. 
(26) Chen, S.; Tam, Y. Y. C.; Lin, P. J. C.; Leung, A. K. K.; Tam, Y. K.; Cullis, P. R. Development of lipid nanoparticle formulations of siRNA for hepatocyte gene silencing following subcutaneous administration. J. Controlled Release 2014, 196, 106-112.

(27) Box, G. E. P.; Behnken, D. W. Some New Three Level Designs for the Study of Quantitative Variables. Technometrics 1960, 2, 455475.

(28) Soema, P. C.; Willems, G.-J.; Jiskoot, W.; Amorij, J.-P.; Kersten, G. F. Predicting the influence of liposomal lipid composition on liposome size, zeta potential and liposome-induced dendritic cell maturation using a design of experiments approach. Eur. J. Pharm. Biopharm. 2015, 94, 427-435.

(29) Jahn, A.; Stavis, S. M.; Hong, J. S.; Vreeland, W. N.; DeVoe, D. L.; Gaitan, M. Microfluidic mixing and the formation of nanoscale lipid vesicles. ACS Nano 2010, 4, 2077-2087.

(30) Zook, J. M.; Vreeland, W. N. Effects of temperature, acyl chain length, and flow-rate ratio on liposome formation and size in a microfluidic hydrodynamic focusing device. Soft Matter 2010, 6, $1352-1360$

(31) Balbino, T. A.; Azzoni, A. R.; de la Torre, L. G. Microfluidic devices for continuous production of $\mathrm{pDNA} /$ cationic liposome complexes for gene delivery and vaccine therapy. Colloids Surf., B 2013, 111, 203-210.

(32) Gasperini, A. A. M.; Puentes-Martinez, X. E.; Balbino, T. A.; de Paula Rigoletto, T.; de Sá Cavalcanti Corrêa, G.; Cassago, A.; Portugal, R. V.; de La Torre, L. G.; Cavalcanti, L. P. Association Between Cationic Liposomes and Low Molecular Weight Hyaluronic Acid. Langmuir 2015, 31, 3308-3317. 